François-Lecompte A. et Prim-Allaz I., 2009, Les français et le tourisme durable, proposition d'une typologie, $1^{\text {ère }}$ journée de recherche ADERSE, La Rochelle, mars.

\title{
Les français et le tourisme durable : proposition d'une typologie $^{3}$
}

\author{
Agnès François-Lecompte * \\ Maître de Conférences \\ Université Bretagne Sud \\ Isabelle Prim-Allaz ** \\ Maître de Conférences \\ Université de Lyon (Coactis/Département Tourisme Lyon 2)
}

* Université Bretagne Sud, IREA (EA4251), 1 rue de la loi, 56000, Vannes, agnes.lecompte@univ-ubs.fr

** Coactis, ISH-Lyon 2 / Département tourisme, 14 avenue Berthelot, 69363 Lyon, Isabelle.Prim-Allaz@univ-lyon2.fr

\footnotetext{
${ }^{3}$ Les auteurs remercient les étudiants du Master 1 Tourisme et Patrimoine de l'université de Lyon 2 pour leur contribution à la collecte des données.
} 


\title{
Les français et le tourisme durable : proposition d'une typologie
}

Résumé : Cet article présente une typologie des consommateurs français en matière de tourisme durable. Les analyses, menées sur un échantillon de 545 individus montrent l'existence de 5 profils de touristes, dont deux sont qualifiés de durables et 3 de non durables. La description des profils obtenus montrent que les individus sensibles au tourisme durable sont aussi des individus qui pratiquent une consommation socialement responsable.

Mots clés : tourisme durable, typologie, consommation socialement responsable

\section{French people and sustainable tourism: proposition of a typology}

\begin{abstract}
This article presents a cluster analysis of French consumers based on sustainable tourism. Analyses, led on a sample of 545 individuals, show the existence of 5 tourists' profiles, among which two are considered as sustainable and 3 as non sustainable. The description of the obtained profiles shows that individuals sensitive to sustainable tourism also have a socially responsible consumption.
\end{abstract}

Key words: sustainable tourism, cluster analysis, socially responsible consumption. 


\section{Les français et le tourisme durable : proposition d'une typologie}

Dans un contexte économique actuellement morose, le tourisme est l'une des rares activités à afficher une croissance régulière. Les données montrent une tendance à la hausse du tourisme international qui semble inéluctable: en 2007, près de 900 millions de voyageurs ont traversé la frontière pour séjourner pour des raisons touristiques dans un autre pays, soit 100 millions de plus qu'en 2005 (Organisation Mondiale du tourisme, 2008, baromètre du tourisme mondial, 2008). Selon les prévisions de l'OMT, ce chiffre devrait atteindre 1,6 milliards en 2020 et fait dire à certains que le tourisme représente l'activité économique la plus prometteuse pour l'humanité (Babou et Callot, 2007). Cependant, si le tourisme offre de belles perspectives économiques, les conditions de son expansion sont sujettes à discussion. Béji-Bécheur et Bensebaa (2006) résument ces menaces en quatre points :

- Sur le plan économique, les revenus sont souvent captés par des intermédiaires et profitent peu aux populations locales. De plus, les emplois dans le secteur touristique sont souvent précaires et saisonniers ;

- Au niveau règlementaire, les règles du droit international restent souvent bafouées (travail des enfants, destruction des structures traditionnelles...);

- Sur le plan éthique, les droits de l'homme ne sont pas toujours respectés (prostitution, transferts de populations, inégalités des droits...);

- Sur le plan culturel, on assiste souvent à une folklorisation des rites et des cultures.

A ces quatre points, nous pouvons ajouter la dimension environnementale. L'impact de l'industrie du tourisme sur l'environnement est en effet préoccupant. A titre d'exemple, cette industrie représente $4 \%$ du PIB mondial mais émet de $4 \%$ à $10 \%$ des gaz à effet de serre de l'économie mondiale, ceci en très grande partie à cause des transports internationaux aériens. Ainsi, 89\% des gaz à effet de serre du tourisme sont liés aux allers et retours entre le lieu de résidence et la destination. (La Revue Durable, juillet-septembre 2008).

De ces différents constats est née l'idée d'un tourisme plus conforme aux principes du développement durable, c'est-à-dire plus juste, plus équitable et davantage respectueux de l'environnement. Depuis les années 1980 , le tourisme durable ${ }^{4}$ s'est peu à peu développé pour devenir ces derniers temps une tendance majeure de ce secteur d'activité.

Du coté institutionnel, une première conférence s'est réunie sur ce thème en 1995 et a donné lieu à une «charte du tourisme durable ». En 1999, l'Organisation Mondiale du Tourisme s'empare à son tour de ce thème et publie avec l'ONU un code mondial d'éthique du tourisme.

Du coté des professionnels, les agences de voyage sont de plus en plus nombreuses à proposer des voyages 'durables' ou 'éthiques'. Les plus avancées d'entre elles sont à l'origine de la création d'un label en 2008, reconnu par l'Etat, 'Agir pour un Tourisme Responsable' et dont les premiers bénéficiaires sont Allibert, Atalante, Chamina Voyages et Voyageurs du Monde. La parution en $2008 \mathrm{du}$ " guide du routard du tourisme durable » constitue un autre signe fort de cette démocratisation auprès du grand public. L'offre de tourisme durable se généralise, semblant donc indiquer qu'il répond à une attente forte des consommateurs.

\footnotetext{
${ }^{4}$ Les termes de tourisme éthique ou responsable sont aussi utilisés dans un sens très proche de celui du tourisme durable. D'autres termes sont parfois privilégiés pour mettre davantage l'accent sur un aspect précis : tourisme solidaire ou équitable en référence au développement des populations d'accueil, tourisme vert ou écotourisme en référence aux critères environnementaux.
} 
Cependant, le degré d'adhésion réel des consommateurs au concept de tourisme durable et la part des individus intéressés par ce type de voyage sont mal connus. Dans le domaine académique, la littérature se consacre essentiellement à des études de cas sur la mise en application des principes du tourisme durable par des destinations touristiques. La demande est souvent considérée comme une variable d'ajustement, c'est-à-dire qui s'adaptera forcément au développement de l'offre (Dolnicar et al., 2008). Des questions simples telles que : «Qui sont les touristes durables ?», "Quelle proportion représentent-ils parmi les français ? », «Que sont-ils prêts changer dans leur façon de voyager? », « Sont-ils des consommateurs comme les autres ? » ne trouvent pas de réponse claire dans la littérature académique ou professionnelle. Pour les acteurs du secteur touristique, il est cependant important de bien cibler les individus les plus à même d'adhérer aux principes et aux contraintes du tourisme durable à la fois pour éviter ainsi des dépenses inutiles vis-à-vis des personnes non intéressées mais aussi pour ne pas faire venir dans un cadre fragile des touristes ne respectant pas les règles de base de la préservation (Treager et al, 1997).

Cet article s'intéresse donc aux attitudes des consommateurs français vis-à-vis du tourisme durable. Plus précisément, nous souhaitons élaborer une typologie afin d'identifier les différents profils de voyageurs, allant des plus enclins à pratiquer un tourisme durable à ceux qui au contraire le rejettent.

La description opérationnelle de ces différents profils de touristes nous amènera à investiguer une question particulière ; à savoir, existe-t-il un lien entre tourisme durable et consommation socialement responsable? Autrement dit, les individus réceptifs au tourisme durable ont-ils plus largement des préoccupations sociales et environnementales qui s'expriment dans leur consommation quotidienne? On peut en effet supposer qu'un individu soucieux du développement durable dans sa façon de voyager le sera également dans ses autres actes d'achat. Ce résultat permettrait le cas échéant de pouvoir cibler les touristes durables, au-delà de leur seul comportement de voyage.

La première partie, consacrée à la revue de la littérature, nous amènera à définir le tourisme durable et à présenter les travaux portant sur les touristes durables. Nous présenterons également la notion de consommation socialement responsable. Enfin, la seconde partie sera consacrée à la présentation de la méthodologie et des résultats.

Il est important de noter que nous adopterons dans cette étude une vision large du tourisme, conformément aux statistiques nationales, à savoir tout déplacement hors de son domicile d'au moins une nuit et pour un motif autre que professionnel ${ }^{5}$. Ceci inclut donc toute forme de vacances passées hors de chez soi, que ce soit en France ou à l'étranger, par l'intermédiaire d'un professionnel du tourisme, agence de voyage, tour opérator ou librement organisés par le consommateur ou son entourage.

\section{Revue de littérature}

\subsection{Définition du tourisme durable (TD)}

Les chercheurs mettent en avant le caractère très large et peu précis du concept de tourisme durable (Liu, 2003, Lansing et De Vries, 2006, Bramwell et Lane, 2008). La définition donnée par l'OMT en 2001 illustre en effet les frontières très lâches de cette notion : le TD doit satisfaire « les besoins actuels des touristes et des régions d'accueil tout en protégeant et en améliorant les perspectives pour l'avenir. Il est vu comme menant à la gestion de toutes les ressources de telle sorte que les besoins économiques, sociaux et esthétiques puissent être

\footnotetext{
${ }^{5}$ Cette définition est reprise de l'Organisation Mondiale du Tourisme.
} 
satisfaits tout en maintenant l'intégrité culturelle, les processus écologiques essentiels, la diversité biologique, et les systèmes vivants ».

Par simplicité, certains auteurs ne se focalisent que sur certains aspects comme par exemple l'effet du voyage sur l'environnement socioculturel et physique de la population d'accueil (Choi et Sirakaya, 2005). Ils excluent de ce fait le transport jusqu'au lieu de séjour qui constitue pourtant le principal poste de pollution du tourisme (Gössling et al., 2008). Dolnicar, Crouch et Long (2008) se focalisent uniquement sur les aspects environnementaux, oubliant l'aspect humain. En réalité, la recherche académique sur le TD semble encore émergente et essentiellement consacrée à des études de cas pratiques.

Pour donner plus de cohérence à ce champs de recherche, Bramwell et Lane (2008) appellent à adopter une vision large du TD englobant toutes les étapes du voyage et ne se réduisant pas aux seuls aspects environnementaux. C'est la posture que nous adopterons pour cette étude. En reprenant les trois piliers du tourisme durable, il est possible d'en résumer les principaux enjeux. L'impact environnemental consiste à éviter tout acte amenant à une pollution de l'eau, de l'air ou du sol ou encore d'une consommation excessive et superflue de ces ressources rares au détriment des populations d'accueil (Lansing et De Vries, 2007). De plus en plus, c'est la pollution engendrée par les transports aériens qui est mise en avant (Sommet de Helsingborg, 2007 ${ }^{6}$ d'après Gössling et al, 2008). Du point de vue économique, l'enjeu principal consiste à éviter les fuites d'argent (Lansing et De Vries, 2007), c'est-à-dire faire en sorte que l'argent dépensé par le touriste revienne en plus grande partie aux populations d'accueil. Cela passe par l'achat de produits locaux plutôt que l'importation des biens et services de l'étranger, ou encore de faire appel à des établissements ou des services possédés par les locaux ou les associant fortement. Concernant le pilier social, il s'agit de préserver le capital socioculturel des populations visitées. Le tourisme de masse provoque souvent en effet des conséquences indésirables pour les communautés d'accueil : hausses des prix, dégradation $\mathrm{du}$ patrimoine naturel ou culturel, perdition des valeurs, des traditions locales, occidentalisation des styles de vie (Lansing et De Vries, 2007). Le tourisme durable est donc présenté comme une alternative au tourisme de conventionnel de masse motivé uniquement par la rentabilité et a pour objectif de contourner les problèmes mentionnés précédemment.

\subsection{Le touriste durable : que sait-on de lui ?}

Les recherches sur le TD portent le plus souvent sur la mise en application des principes de 'durabilité' sur les destination touristiques, se focalisant ainsi sur le coté 'offre' du marché plutôt que sur les consommateurs (Dolcinar et al., 2008). Dans ces différentes études de cas, différentes segmentation des touristes en matière de TD ont été proposées (travaux de Palacio et McCool, 1997, Galloway, 2002, Weaver et Lawton, 2002). Ces travaux sont cependant difficilement généralisables car construits dans des cas d'application bien précis. Dolnicar, Crouch et Long (2008) proposent une méta-analyse des recherches qualifiant les touristes sensibles à l'environnement (environment-friendly tourists). Même si elle n'aborde qu'une seule des dimensions du tourisme durable, cette étude n'en demeure pas moins riche d'enseignements. La conclusion des auteurs est que peu de caractéristiques individuelles ont finalement été étudiées dans la littérature existante à ce jour, concluant que ce que l'on sait finalement sur les touristes 'pro-environnementaux' est qu'ils sont plutôt éduqués, avec des revenus élevés et en quête d'apprentissage. L'âge ne donne pas de résultats, le sexe a été trop peu étudié. Une des caractéristiques proposées par Dolnicar, Crouch et Long (2008) pour

\footnotetext{
${ }^{6}$ En septembre 2007 a eu lieu une rencontre international de 35 experts sur le tourisme durable à Helsingborg en Suède. A partir des données concernant les changements climatiques et l'augmentation des flux touristiques, ils ont proposé un agenda de recherche sur le tourisme durable et émis des recommandations quant aux actions qui pourraient être entreprises par l'industrie et les gouvernements.
} 
identifier les touristes durables est l'acceptation de renoncer à son confort quotidien. Becker et al. (1981) ont, en effet, montré qu'il existe un arbitrage (trade-off) entre le fait d'accepter une diminution de son confort et les préoccupations environnementales. Cet arbitrage est probablement encore plus sévère en matière touristique dans la mesure où il s'agit d'un contexte étroitement lié à la notion de plaisir et non pas à celle de sacrifice. La piste de recherche proposée par Dolnicar, Crouch et Long (2008) n'a cependant pas été testée.

Quelques éléments de connaissances sur le touriste durable peuvent être tirés des résultats d'enquête. Une enquête TNS-Sofres réalisée en mars 2008 auprès de 800 individus s'intéresse aux représentations des consommateurs en matière de tourisme responsable. Il en ressort que les français connaissent mal ce concept, s'en font une idée pas toujours exacte mais sont intéressés dans leur grande majorité par cette nouvelle façon de voyager. Une autre enquête réalisée en 2004 par l'UNAT ${ }^{7}$ pour le compte du ministère des affaires étrangères a étudié les images, attentes et freins des voyageurs français à l'égard du tourisme solidaire. Il se dégage que les personnes intéressées appartiennent à des CSP +, ont un niveau d'étude plus élevé que la moyenne et appartiennent souvent à une association. Ils voyagent plus que la moyenne et privilégient des voyages de découverte des populations et des cultures plus que des séjours type «club de détente au bord de la mer». Ces résultats sont intéressants et méritent d'être validé pour le concept plus large de tourisme durable.

Au final, les travaux académiques ou institutionnels ont adopté des visions assez variées du TD, rendant les résultats peu comparables. Nous nous proposons donc dans la suite de cet article de bâtir une typologie cherchant à mieux comprendre qui est susceptible de pratiquer cette nouvelle forme de voyage, sous quelle forme, et d'étudier le lien entre TD et consommation socialement responsable (CSR).

\subsection{Le touriste durable : un consommateur socialement responsable ?}

Dans une vision large, la CSR regroupe tous les comportements de consommation où l'individu prend en compte les conséquences publiques de sa consommation privée et utilise son pouvoir d'achat pour induire des changements dans la société (Webster, 1975). Les acheteurs responsables agissent au-delà de leurs simples intérêts et tiennent compte de l'impact de leur consommation sur l'environnement physique et/ou sur différentes catégories de personnes. Cela inclut l'achat selon le degré de responsabilité des entreprises, mais aussi d'autres comportements engagés comme par exemple, le fait de privilégier des produits français, ou au contraire de boycotter certains pays.

Une des questions que nous nous posons dans cet article est de savoir s'il existe un lien entre TD et CSR. Pour cela, nous nous baserons sur les travaux de François-Lecompte \& ValetteFlorence (2006), travaux qui présentent l'intérêt d'être à la fois récents et conduits dans un contexte français.

D'après ces auteurs, la CSR peut se résumer à 5 facteurs :

- Le refus d'acheter aux entreprises dont le comportement est jugé «irresponsable » comme par exemple le fait de faire travailler des enfants, de maltraiter ses salariés ou de polluer l'environnement.

- L'intention d'acheter des produits dont une partie du prix revient à une cause déterminée (Achat produit-partage, de produits issus du commerce équitable)

- La volonté d'aider les petits commerces et de défendre ainsi l'emploi local, un lien de proximité, et la diversité du paysage commercial.

\footnotetext{
${ }^{7}$ Union Nationale des Associations de Tourisme et de Plein Air, étude sur échantillon de 402 individus de plus de 18 ans, novembre 2004
} 
- La sensibilité à l'origine des produits en privilégiant dans ses achats sa région d'origine, les produits français voir européens.

- La volonté de réduire son volume de consommation à ce qui est réellement nécessaire. Cela consiste à ne pas trop consommer et tenter faire certaines choses soi-même plutôt que de les acheter.

Le lien entre TD et CSR n'a, semble-t-il, jamais été testé. On peut pourtant supposer que ces deux comportements sont l'expression des mêmes valeurs chez le consommateur. Il parait difficile d'imaginer qu'un individu soucieux d'environnement dans ses achats quotidiens ne le soit pas également un minimum dans ses comportements touristiques.

Les trois premières facettes de la CSR, considérée dans un contexte touristique, aboutissent à l'idée de choisir des intermédiaires ayant un comportement responsable y compris vis-à-vis de leurs collaborateurs, garantissant par exemple un voyage écologique et solidaire avec les populations d'accueil. Les offres des voyagistes tels qu'Atalante, Alibert et plus largement tous ceux associés au nouveau label ATR se positionnent exactement dans cette perspective.

La quatrième facette de la CSR conduirait en matière de tourisme à privilégier les voyages de proximité, près de chez soi afin de faire vivre la culture et les emplois régionaux. C'est l'idée même du tourisme de proximité, qui apparaît de plus en plus comme l'avenir du TD.

Enfin, la cinquième facette de la CSR, appliquée au tourisme consisterait à voyager moins, sans intermédiaire et peut-être de façon plus proche de la nature. Cette idée d'un tourisme moins polluant, plus simple est certainement elle aussi liée au concept de TD.

Le rapprochement des définitions de TD et de la CSR montre qu'il existe de nombreuses proximités entre les deux concepts. Le tourisme n'étant qu'un secteur particulier, il apparaîtrait naturel que les consommateurs y exercent leur préoccupations sociales et environnementales, tout autant que dans les autres univers de consommation.

Une limite cependant pourrait résider dans la spécificité des dépenses touristiques. En effet, comme le rappellent Evrard, Bourgeon et Petr (2000) les activités de loisirs ont trois fonctions principales : le développement personnel, le divertissement et le délassement. Le tourisme est donc une consommation avant tout expérientielle s'adressant aux dimensions hédoniques et affectives du consommateur, a priori à l'opposé de la notion d'effort et de sacrifice. Or, la CSR amène à devoir renoncer, au moins en partie à son plaisir personnel. Les vacances seraient-elles, dans cette vision, la seule bouffée d'oxygène que le consommateur responsable s'autorise, oubliant toutes les contraintes avec son seul plaisir en tête?

Les sections suivantes aborderont la démarche méthodologique adoptée ainsi que les principaux résultats obtenus.

\section{Elaboration d'une typologie du touriste durable}

\subsection{Méthodologie et présentation de l'échantillon}

Cette étude a été menée à l'aide d'un questionnaire, comprenant plus d'une centaine de questions. Trois sections ont, entre autres, été introduites dans le questionnaire pour mesurer (1) l'échelle de mesure de la CSR proposée par François-Lecompte (2005), (2) les pratiques des répondants relatives au tourisme en général et au tourisme durable en particulier et (3) le profil socio-démographique des répondants.

Concernant la mesure des pratiques de tourisme durable (point 2), nous avons généré une batterie d'items en nous inspirant de la littérature à la fois académique, institutionnelle et professionnelle sur le tourisme durable. Nous avons veillé à représenter les trois dimensions 
du tourisme durable: bonne répartition économique des revenus du tourisme, respect de l'environnement à toutes les étapes du voyage, respect du capital socioculturel du lieu visité. Concernant le pilier environnemental, plusieurs items avaient pour vocation de mesurer à quel point le répondant est prêt à renoncer à des voyages lointains et donc polluants à cause du transport aérien et à privilégier en conséquence un tourisme de proximité, identifié de plus en plus comme la solution du tourisme durable (la Revue Durable, oct-nov 2008). Les items incluaient également des comportements liés à l'acceptation de renoncer au confort matériel pendant le voyage, suivant ainsi la proposition de Dolnicar, Crouch et Long (2008). La liste de comportements, censés traduire le tourisme durable, a été validée et complétée par un groupe d'étudiants en Master tourisme de façon à s'assurer que toutes les facettes du concept étaient bien mesurées dans le questionnaire. En tout, vingt-cinq items ont été retenus (présentés en tableau 1).

Le questionnaire a été administré par deux canaux : (1) administration en face-à-face ; (2) administration par Internet. Les questionnaires ont été collectés par un groupe de 50 étudiants en Tourisme auxquels avaient été donnés des quotas en termes de sexe et d'âge (sur la base des données INSEE).

In fine, 545 questionnaires ont été collectés, dont 49, 4\% en face-à-face et 50,6\% via Internet. L'échantillon comprend 47,2\% d'hommes et 52,8\% de femmes. 55,6\% des répondants ont des enfants. L'âge moyen est de 42,48 ans (médiane 42 ans et écart-type de 16,4 ans). 74, $1 \%$ de l'échantillon habite en zone urbaine ou péri-urbaine. La répartition des revenus se rapproche de la moyenne française. L'échantillon est également varié en termes de niveau d'étude et de CSP.

\subsection{Présentation des 5 facteurs de tourisme durable}

Dans un premier temps, nous avons conduit une analyse factorielle afin d'explorer les formes de voyage durable qui apparaissent derrière les 25 items retenus pour mesurer les comportements des répondants en matière de tourisme durable et d'identifier les facettes les mieux accueillies par le grand public. Une ACP a donc été menée et après suppression de 7 items ayant une qualité de représentation inférieure à 0,5 , il ressort de cette analyse une structure en 5 facteurs, permettant de restituer $61 \%$ de variance (Cf. Tableau 1).

Ces cinq facteurs peuvent s'interpréter de la façon suivante ${ }^{8}$. :

- Accepter de renoncer au confort (13,05\% de variance)

- Recourir à un voyagiste responsable $(12,15 \%$ de variance)

- Maximiser les ressources des locaux (11,99\% de variance)

- Aller moins loin $(11,91 \%$ de variance)

- Protéger le capital social, culturel et naturel des zones visitées (11,73\% de variance)

\footnotetext{
${ }^{8}$ Pour les 18 items, les poids factoriels sont tous supérieurs ou égaux à 0,5 , de même que les qualités de représentation. Les items sont présentés par ordre de contribution décroissante au facteur.
} 
Tableau 1 -Résultat de l'analyse factorielle sur les 25 items de tourisme durable, après rotation Varimax $(n=545)$

\begin{tabular}{|c|c|c|c|c|c|c|}
\hline & & \multicolumn{5}{|c|}{ Composante } \\
\hline & & 1 & 2 & 3 & 4 & 5 \\
\hline \multirow{4}{*}{$\begin{array}{l}\text { Accepter de renoncer } \\
\text { au confort }\end{array}$} & $\begin{array}{l}\text { Eviter de loger dans des hôtels luxueux dans } \\
\text { des pays en voie de développement }\end{array}$ & 0,819 & & & & \\
\hline & $\begin{array}{l}\text { Loger chez l'habitant ou dans des hôtels } \\
\text { tenus par des locaux plutôt que dans grandes } \\
\text { chaînes hôtelières }\end{array}$ & 0,681 & & & & \\
\hline & $\begin{array}{l}\text { Accepter de rogner sur les conditions de } \\
\text { confort lors de ses voyages (hébergement, } \\
\text { alimentation et transport aux conditions } \\
\text { locales) }\end{array}$ & 0,663 & & & & \\
\hline & Eviter des voyages de type croisière & 0,645 & & & & \\
\hline \multirow{3}{*}{$\begin{array}{l}\text { Recourir à un } \\
\text { voyagiste responsable }\end{array}$} & $\begin{array}{l}\text { Partir avec des organisateurs de voyage qui } \\
\text { garantissent le respect de l'environnement } \\
\text { des lieux visités }\end{array}$ & & 0,810 & & & \\
\hline & $\begin{array}{l}\text { Privilégier des organisateurs de voyage qui } \\
\text { financent des projets de développement au } \\
\text { profit des populations locales }\end{array}$ & & 0,800 & & & \\
\hline & $\begin{array}{l}\text { S'assurer que les entreprises touristiques } \\
\text { respectent les règles internationales du droit } \\
\text { du travail }\end{array}$ & & 0,724 & & & \\
\hline \multirow{4}{*}{$\begin{array}{l}\text { Maximiser les } \\
\text { ressources des locaux }\end{array}$} & Se sentir utile au pays visité & & & 0,681 & & \\
\hline & $\begin{array}{l}\text { Vivre comme les locaux pour ne pas gaspiller } \\
\text { leurs ressources }\end{array}$ & & & 0,629 & & \\
\hline & $\begin{array}{l}\text { S'assurer qu'une part conséquente des } \\
\text { revenus générés par votre voyage revient aux } \\
\text { pays visités }\end{array}$ & & & 0,612 & & \\
\hline & Economiser les ressources locales rares & & & 0,485 & & \\
\hline \multirow{3}{*}{ Aller moins loin } & Eviter de voyager à l'autre bout du monde & & & & 0,849 & \\
\hline & Privilégier des séjours près de chez soi & & & & 0,827 & \\
\hline & Eviter de prendre l'avion pour se déplacer & & & & 0,748 & \\
\hline \multirow{4}{*}{$\begin{array}{l}\text { Protéger le capital des } \\
\text { zones visitées }\end{array}$} & $\begin{array}{l}\text { Faire travailler le commerce et l'artisanat des } \\
\text { populations visitées }\end{array}$ & & & & & 0,764 \\
\hline & Ne pas dégrader les sites naturels visités & & & & & 0,720 \\
\hline & Respecter les valeurs des populations visitées & & & & & 0,669 \\
\hline & $\begin{array}{l}\text { Avoir le maximum d'échanges avec les } \\
\text { populations des lieux visités }\end{array}$ & & & & & 0,518 \\
\hline
\end{tabular}

\subsection{Présentation de la typologie}

A partir des résultats obtenus par l'analyse factorielle, des profils d'individus en matière de tourisme durable ont été établis. Une analyse ascendante hiérarchique a été menée sur les scores des individus aux 5 facteurs. La chaîne des agrégations présente un palier dans la qualité de la typologie entre la solution à 5 groupes et celle à 6 groupes ${ }^{9}$. L'analyse par les nuées dynamiques montre de plus qu'une structure à 5 groupes présente des effectifs équilibrés et une bonne validité opérationnelle conformément aux recommandations de Jolibert et Jourdan (2006). Les profils des 5 groupes sont présentés dans le tableau 2.

\footnotetext{
${ }^{9}$ Méthode décrite dans Tenenhaus (2007).
} 
Tableau 2 - Typologie ${ }^{8}$

\begin{tabular}{|c|c|c|c|c|c|}
\hline & Les néo-durables & Les campeurs & Les séjour-clubs & Les institutionnels cultureux & Les durables d'aventure \\
\hline Effectifs & 112 & 98 & 64 & 83 & 136 \\
\hline $\begin{array}{l}\text { Renoncer au confort } \\
\text { valeur moyenne }\end{array}$ & $\begin{array}{l}\text { Neutre } \\
0,10\end{array}$ & $\begin{array}{l}\text { Prêt à faire qq efforts en } \\
\text { terme de confort } \\
0,39\end{array}$ & $\begin{array}{l}\text { Pas prêt à renoncer à } \\
\text { son confort } \\
-0,58\end{array}$ & $\begin{array}{l}\text { Pas du tout prêt à renoncer à son } \\
\text { confort } \\
-1,32\end{array}$ & $\begin{array}{l}\text { Les plus à même de renoncer à } \\
\text { leur confort } \\
0,71\end{array}$ \\
\hline $\begin{array}{l}\text { Voyagiste responsable } \\
\text { valeur moyenne }\end{array}$ & $\begin{array}{l}\text { Pas prêts } \\
-0,37\end{array}$ & $\begin{array}{l}\text { Neutre - } \\
-0,11\end{array}$ & $\begin{array}{l}\text { Neutre - } \\
-0,17\end{array}$ & $\begin{array}{l}\text { Prêts } \\
0,29\end{array}$ & $\begin{array}{l}\text { Prêts } \\
0,29\end{array}$ \\
\hline $\begin{array}{l}\text { Ressources des locaux } \\
\text { (protection des ressources } \\
\text { locales) valeur moyenne }\end{array}$ & $\begin{array}{l}\text { Assez concerné } \\
0,57\end{array}$ & $\begin{array}{l}\text { Pas concerné } \\
-1,064\end{array}$ & $\begin{array}{l}\text { Assez concerné } \\
0,50\end{array}$ & $\begin{array}{l}\text { pas concerné } \\
-0,44\end{array}$ & $\begin{array}{l}\text { Assez concerné } \\
0,33\end{array}$ \\
\hline $\begin{array}{l}\text { Aller moins loin } \\
\text { valeur moyenne }\end{array}$ & $\begin{array}{l}\text { Tout à fait prêt } \\
0,96\end{array}$ & $\begin{array}{l}\text { Assez prêt } \\
0,31\end{array}$ & $\begin{array}{l}\text { Neutre } \\
-0,036\end{array}$ & $\begin{array}{l}\text { Pas prêt } \\
-0,47\end{array}$ & $\begin{array}{l}\text { pas prêt } \\
-0,70\end{array}$ \\
\hline $\begin{array}{l}\text { Protection du capital social } \\
\text { et culturel valeur moyenne }\end{array}$ & $\begin{array}{l}\text { Assez préoccupé } \\
0,52\end{array}$ & $\begin{array}{l}\text { Peu concernés } \\
-0,44\end{array}$ & $\begin{array}{l}\text { Pas du tout concerné } \\
-1,73\end{array}$ & $\begin{array}{l}\text { Assez préoccupé } \\
0,65\end{array}$ & $\begin{array}{l}\text { Assez préoccupé } \\
0,30\end{array}$ \\
\hline
\end{tabular}

Tableau 3 - Habitudes de voyage

\begin{tabular}{|c|c|c|c|c|c|}
\hline & Les néo-durables & Les campeurs & Les séjour-clubs & Les institutionnels cultureux & Les durables d'aventure \\
\hline $\begin{array}{l}\text { Détente } \\
\text { Anova, } \mathrm{p}=0,071, \mathrm{moy}=3,24\end{array}$ & 3,25 & 3,30 & $\begin{array}{l}\text { Recherche détente } \\
\mathbf{3 , 3 8}\end{array}$ & 3,29 & $\begin{array}{l}\text { Ne recherche pas détente } \\
\mathbf{3 , 1 0}\end{array}$ \\
\hline $\begin{array}{l}\text { Culture } \\
\text { Anova, } p=0,001, \text { moy }=3,00\end{array}$ & 2,99 & $\begin{array}{l}\text { Ne rech pas culture } \\
\mathbf{2 , 8 4}\end{array}$ & $\begin{array}{l}\text { Ne rech pas culture } \\
\mathbf{2 , 8 2}\end{array}$ & $\begin{array}{l}\text { Moy sur rech culture }\left(2^{\text {nd }}\right. \\
\text { score mais pas signif.) } 3,00\end{array}$ & $\begin{array}{l}\text { Rech culture } \\
3,19\end{array}$ \\
\hline $\begin{array}{l}\text { Aventure } \\
\text { Anova, } p=0,000, \text { moy }=2,46\end{array}$ & $\begin{array}{l}\text { Ne rech pas aventure } \\
\mathbf{2 , 3 8}\end{array}$ & $\begin{array}{l}\text { Ne rech pas aventure } \\
\mathbf{2 , 2 9}\end{array}$ & $\begin{array}{l}\text { Rech aventure ++ } \\
2,72\end{array}$ & $\begin{array}{l}\text { Ne rech pas aventure } \\
\mathbf{2 , 2 4}\end{array}$ & $\begin{array}{l}\text { Rech aventure } \\
2,66\end{array}$ \\
\hline $\begin{array}{l}\text { Sport } \\
\text { Anova, } \mathrm{p}=0,002 ; \mathrm{moy}=2,16\end{array}$ & 2,21 & 2,15 & 2,35 & $\begin{array}{l}\text { Ne rech pas sport } \\
\mathbf{1 , 8 1}\end{array}$ & 2,23 \\
\hline $\begin{array}{l}\text { Rencontres } \\
\text { Anova, } \mathrm{p}=0,000 ; \mathrm{moy}=3,01\end{array}$ & $\begin{array}{l}\text { Rech rencontres } \\
\mathbf{3 , 0 9}\end{array}$ & $\begin{array}{l}\text { Ne rech pas rencontres } \\
\mathbf{2 , 7 6}\end{array}$ & $\begin{array}{l}\text { Ne rech pas rencontres } \\
\mathbf{2 , 8 6}\end{array}$ & $\begin{array}{l}\text { Ne rech pas rencontres } \\
\mathbf{2 , 8 3}\end{array}$ & $\begin{array}{l}\text { Rech rencontres } \\
\mathbf{3 , 2 9}\end{array}$ \\
\hline $\begin{array}{l}\text { Voyages organisés } \\
\text { Anova } \mathrm{p}=0,000, \mathrm{moy}=1,78\end{array}$ & $\begin{array}{l}\text { Ne rech pas voyages org. } \\
\mathbf{1 , 6 8}\end{array}$ & $\begin{array}{l}\text { Ne rech pas voyages org. } \\
\mathbf{1 , 7 2}\end{array}$ & $\begin{array}{l}\text { Voyage organisé } \\
1,97\end{array}$ & $\begin{array}{l}\text { Voyage organisé } \\
2,20\end{array}$ & $\begin{array}{l}\text { Ne rech pas voyages org. } \\
\mathbf{1 , 5 8}\end{array}$ \\
\hline $\begin{array}{l}\text { Voy. Org. par soi-même } \\
\text { Anova } p=0,000, \text { moy }=3,23\end{array}$ & 3,25 & $\begin{array}{l}\text { Non } \\
\mathbf{3 , 1 7}\end{array}$ & $\begin{array}{l}\text { Non } \\
\mathbf{3 , 0 8} \\
\end{array}$ & $\begin{array}{l}\text { Non } \\
\mathbf{3 , 0 0} \\
\end{array}$ & $\begin{array}{l}\text { Oui } \\
3,46\end{array}$ \\
\hline $\begin{array}{l}\text { Voyage groupe amis } \\
\text { Anova } \mathrm{p}=0,000, \mathrm{moy}=2,56\end{array}$ & $\begin{array}{l}\text { Non } \\
\mathbf{2 , 4 1}\end{array}$ & 2,61 & $\begin{array}{l}\text { oui } \\
2,77\end{array}$ & $\begin{array}{ll}\text { non } \\
\mathbf{2 , 2 8}\end{array}$ & $\begin{array}{l}\text { oui } \\
2,71\end{array}$ \\
\hline
\end{tabular}

\footnotetext{
${ }^{8}$ Les scores des individus sont centrés réduits. Un score proche de 0 montre que le groupe est neutre sur la dimension, un score positif $=$ groupe ayant une moyenne supérieure sur la dimension (score négatif = moyenne inférieure)
} 


\begin{tabular}{|c|c|c|c|c|c|}
\hline $\begin{array}{l}\text { Hôtel/bateau } \\
\text { Anova } \mathrm{p}=0,000 ; 2,31\end{array}$ & 2,20 & 2,11 & 2,34 & 2,83 & 2,20 \\
\hline $\begin{array}{l}\text { Location } \\
\text { Anova p=N.s. ; } 2,42\end{array}$ & 2,47 & 2,47 & 2,50 & 2,26 & 2,40 \\
\hline $\begin{array}{l}\text { Famille/amis } \\
\text { Anova } p=0,030 ; 2,49\end{array}$ & 2,64 & 2,48 & 2,54 & 2,26 & 2,50 \\
\hline $\begin{array}{l}\text { Camping/caravane Anova } \\
\mathrm{p}=0,001 ; 1,83\end{array}$ & 1,70 & 2,04 & 1,84 & 1,53 & 1,95 \\
\hline $\begin{array}{l}\text { Utilise voiture } \\
\text { Anova, } \mathrm{p}=0,018, \mathrm{moy}=3,01\end{array}$ & 3,12 & 2,98 & $\begin{array}{l}\text { non } \\
2,87\end{array}$ & $\begin{array}{l}\text { oui } \\
3,21\end{array}$ & $\begin{array}{l}\text { non } \\
2,87\end{array}$ \\
\hline $\begin{array}{l}\text { Utilise train } \\
\text { Anova, } p=0,006, \text { moy }=2,33\end{array}$ & $\begin{array}{l}\text { Non } \\
\mathbf{2 , 1 8} \\
\end{array}$ & 2,25 & 2,48 & $\begin{array}{ll}\text { non } \\
\mathbf{2 , 1 8}\end{array}$ & $\begin{array}{l}\text { oui } \\
2,52 \\
\end{array}$ \\
\hline $\begin{array}{l}\text { Utilise avion } \\
\text { Anova } \mathrm{p}=0,000, \mathrm{moy}=1,72\end{array}$ & $\begin{array}{l}\text { Non } \\
\mathbf{1 , 4 4}\end{array}$ & $\begin{array}{l}\text { non } \\
1,59\end{array}$ & $\begin{array}{l}\text { OUI (bcp plus que les } \\
\text { autres) } 2,16\end{array}$ & 1,70 & $\begin{array}{l}\text { oui } \\
1,82\end{array}$ \\
\hline $\begin{array}{l}\text { Durée séjours touristi/an } \\
\text { En nb de semaines } \\
\mathrm{P}=0,002\end{array}$ & 3,10 & 3,46 & 3,38 & 3,44 & 4,16 \\
\hline $\begin{array}{l}\text { Budget } 1 \text { semaine } \\
\text { vacances étranger (en } \\
\text { euros) } \\
\text { Moy }=724,79\end{array}$ & 652,79 & 660,37 & 749,15 & 915,58 & 720,41 \\
\hline $\begin{array}{l}\text { France/étranger Anova } \\
(p=0,000 ; \text { moy }=-1,20)\end{array}$ & $-1,58$ & $-2,55$ & $-1,48$ & $-0,90$ & 0,04 \\
\hline $\begin{array}{l}\text { Europe /hors Europe } \\
\text { Anova }(\mathrm{p}=0,000 ; \text { moy=- } \\
2,01)\end{array}$ & $-2,39$ & $-3,52$ & $-2,10$ & -.97 & $-1,30$ \\
\hline
\end{tabular}


Le premier groupe d'individus $(\mathrm{n}=112)$ se distingue des autres sur la dimension «aller moins loin ». Il représente des individus sensibles aux ressources économiques et socioculturelles des populations visitées et prêts à pratiquer un tourisme de proximité. Nous les appelons les «néodurables». Le second groupe $(\mathrm{n}=98)$, que nous qualifierons de « campeurs» présentent un profil peu durable; ils sont cependant prêts à aller moins loin et à renoncer un peu à leur confort. Les individus du troisième groupe $(n=64)$, qualifiés de « séjours-clubs » n'entendent pas renoncer à leur confort et se démarquent des autres groupes car ils se soucient peu du patrimoine socioculturel et naturel du lieu visité. Le quatrième groupe $(n=83)$, les « institutionnels cultureux ${ }^{9}$ » sont soucieux du respect du patrimoine socioculturel et naturel du lieu visité et sont intéressés par des voyagistes responsables; ils ne sont cependant prêts à faire aucune concession en matière de confort et de destination. Le dernier groupe $(n=136)$ les "durables d'aventure », sont très sensibles au TD, mais le pratiquent sur des destinations lointaines.

Une fois les groupes définis, nous avons tenté de les décrire au travers d'un certain nombre de variables liées à leurs pratiques de voyage actuelles. Les groupes ont été comparés sur l'ensemble des éléments suivants (Cf. Tableau 3):

- objectif recherché d'un séjour touristique: détente, culture, aventure, sport ou rencontres avec les habitants du lieu visité

- formule privilégiée pour voyager : voyages organisés, voyages organisés par soi-même

- hébergement habituel: hôtel (ou bateau de croisière), location/ gîte, famille/ amis, camping/caravane

- transport utilisé : voiture, train, avion

- durée des séjours touristiques, budget consacré à un séjour d'une semaine à l'étranger

- destination ${ }^{10}$ : France versus l'étranger et Europe versus hors de l'Europe. Des chiffres négatifs indiquent des vacances passées en majorité en France plutôt qu'à l'étranger, et en Europe plutôt que hors Europe. Plus les valeurs sont proches de zéro ou positives, plus c'est l'inverse.

La plupart des analyses présentées dans ces tableaux repose sur des ANOVA. Afin de mesurer la significativité des différences entre les groupes pris deux à deux, le test de Bonferroni a été retenu. Les valeurs en caractère gras indiquent les groupes ayant un niveau significativement inférieur aux autres groupes et celles en caractère gras italique indiquent les groupes ayant un niveau significativement supérieur aux autres groupes.

Le groupe des «néodurables » se caractérise par un budget vacances serré par rapport aux autres groupes. Ces individus ont un intérêt pour des voyages axés sur les rencontres, une réticence à voyager entre amis ou avec des agences. Ils privilégient le logement chez des amis aux autres formules d'hébergement. Ils partent moins longtemps et plutôt en France.

Le groupe des «campeurs » dépense lui aussi de façon mesurée pendant ses vacances. Il voyage simplement, sans intermédiaire. Il loge de préférence en camping et part lui aussi près de chez lui (presque jamais à l'étranger), sans prendre l'avion. Il ne recherche ni la culture, ni l'aventure ni les rencontres et préfère organiser lui-même son voyage. Les « séjours-club » ont été appelés du fait de leur recherche de détente et de dépaysement plus que de culture. Ils apprécient les voyages organisés et partir loin en avion. Ils sont prêts à dépenser 750 euros en moyenne pour une semaine de vacances à l'étranger. Le plaisir est sans doute le maître mot de

\footnotetext{
${ }^{9}$ Le nom «institutionnels » est donné en référence à la typologie de Cohen (1972) in Frochot et Legohérel (2007).

${ }^{10}$ Pour les variables du $3^{\text {ème }}$ bloc (objectifs, formules, hébergement et transport), le répondant devait indiquer ses habitudes ou ses préférences sur une échelle allant de 1 à 4 (1=jamais et 4=toujours). La durée des séjours était exprimé sur une échelle à 6 modalités ( $1=$ moins 2 semaines/ an et $6=+$ de 10 semaines $/$ an). Enfin, concernant la destination, le répondant devait se positionner sur une échelle allant de 1 (100\% en France ou $100 \%$ en Europe) à 10 (100\% à l'étranger ou 100\% hors Europe).
} 
leurs vacances. Les «institutionnels cultureux» ont également recours aux voyages organisés et disposent d'un budget pour leur vacances supérieur aux autres groupes. Ils sont attachés à leur confort et privilégient souvent l'hôtel. Ces individus ne croient pas au concept de tourisme durable, et le considèrent comme une utopie et ne sont logiquement pas prêts à dépenser plus pour ce concept. Ils partent fréquemment hors Europe et recherchent plutôt la culture dans leur voyage. Enfin, les «touristes d'aventure» sont composés d'individus qui partent loin et longtemps en vacances. Ils organisent leurs voyages par eux-mêmes et sont avides de rencontres, de culture et d'expériences fortes plutôt que de détente. Le confort n'est pas important pour eux, ce qui les amène à dormir parfois au camping. Ils sont très réceptifs au concept de TD et ont un profil positif sur les différentes facettes, excepté sur la dimension «aller moins loin ».

Au final, parmi les 5 groupes, 2 ont des profils de touristes durables : le premier est durable, dispose d'un faible budget pour partir en vacances et privilégie des vacances de proximité en famille ou entre amis; le groupe 5 dispose d'un budget plus conséquent et est attentif au TD, au détail près qu'il traverse la planète pour partir en vacances. Les 3 autres groupes sont pas ou peu durables dans leur façon de voyager : le groupe 2 (les campeurs) par manque d'intérêt pour les populations visitées, le groupe 3 (les séjours-clubs) par une priorité donnée à la détente et enfin le groupe 4 (les institutionnels cultureux), dans une moindre mesure, par refus de renoncer au confort et par conviction.

\subsection{Tourisme durable et CSR : quels liens ?}

Afin d'identifier les liens entre tourisme durable et consommation responsable, nous avons dans un second temps cherché à typer les 5 profils de touristes sur la CSR. Dans une première étape, nous avons mené une analyse factorielle sur les 20 items de l'échelle de FrancoisLecompte et Valette-Florence (2006), ce qui nous a permis de retrouver les 5 facteurs de la CSR, à savoir :

- Ne pas acheter aux entreprises jugées irresponsables ;

- Acheter des produits partage ;

- $\quad$ Favoriser les petits commerces ;

- $\quad$ Etre vigilant à l'origine du produit (région, France, Europe) ;

- Réduire son volume de consommation (simplicité volontaire).

Les scores factoriels pour chacune des dimensions de la CSR ont été croisés avec les différents profils de touristes (durables et non durables), à l'aide d'analyse de variance. Le tableau 4 indique les moyennes des 5 sous-groupes sur les 5 facettes de la CSR, le résultat du test de Fisher ainsi que les écarts significatifs entre groupes au regard du test post hoc de Bonferroni. De plus, une simple somme des scores de chaque profil sur les 5 facettes a été calculée afin de faciliter les comparaisons globales.

Dans un premier temps, il est important de remarquer que pour chacune des 5 facettes de la CSR, les ANOVA sont significatives. Les 5 groupes de touristes durables diffèrent donc en termes de CSR, ce qui confirme l'hypothèse de travail adoptée dans ce papier. TD et CSR sont donc des concepts liés. En regardant dans le détail, il apparaît que les deux profils durables, «les néodurables » et les «durables d'aventure » ont un score systématiquement plus élevé que les autres groupes sur chacune des cinq dimensions de la CSR. A l'inverse, les trois profils non durables ont des scores inférieurs à la moyenne ou nuls sur la CSR. Cette démarcation entre touristes durables et non durables se retrouve également dans le score global des individus. Ce premier résultat montre qu'a priori un touriste durable est un consommateur responsable et qu'a contrario un touriste non durable n'est pas non plus un 
consommateur responsable. Ce fonctionnement accrédite l'hypothèse selon laquelle le tourisme n'est qu'un domaine d'activité parmi d'autres et que l'individu peut y appliquer les mêmes critères de choix que dans les autres sphères de consommation. Bien que les voyages soient l'occasion de détente et divertissement, le consommateur ne change pas de comportement à cette occasion, il reste naturel, fidèle à ses critères d'achats habituels, qu'ils soient durables ou non.

$\mathrm{Si}$ on cherche maintenant à mieux caractériser les profils de touristes, nous pouvons cerner deux nuances entre le groupe des «néodurables » et des «durables d'aventure ». Les deux dimensions de la CSR sur lesquelles ils se différencient sont la dimension «entreprise irresponsable» et «origine géographique». Sur la première dimension «entreprise irresponsable », le groupe des «néodurables» ne se différencient pas des touristes non durables. Ce résultat n'est pas surprenant dans la mesure où les «néodurables » ne font pas appel aux organisateurs de voyages : ils s'intéressent donc peu aux entreprises et à leur comportement plus ou moins condamnables, contrairement aux «durables d'aventure». Ceux-ci font appel si besoin à intermédiaire dans leur voyage, mais surtout ils s'intéressent aux pratiques des organisations en général.

Sur la seconde dimension « origine géographique », ce sont les «durables d'aventure » qui ne se différencient pas. Ces touristes privilégiant la découverte de contrées lointaines, et ne sont pas attachés à leur terre d'origine. Les «néodurables » eux sont plus ancrés sur leur territoire et partent donc moins loin en vacances.

Remarquons également que les «campeurs » ont des scores faiblement négatifs sur la CSR ; nous avançons ici l'hypothèse que, comme pour le tourisme, ils s'interrogent peu sur les questions éthiques dans leurs achats. Les « séjours-club » sont définitivement des épicuriens, peu portés sur les aspects d'écologie et d'équité sociale. Enfin, les «institutionnels cultureux » semblent sceptiques face aux concepts de TD comme de CSR.

Tableau 4 : Tourisme durable et consommation socialement responsable

\begin{tabular}{|l|c|c|c|c|c|}
\hline & $\begin{array}{c}\text { Néo- } \\
\text { durables }\end{array}$ & Campeurs & $\begin{array}{c}\text { Séjours- } \\
\text { club }\end{array}$ & $\begin{array}{c}\text { Institutionnels } \\
\text { cultureux }\end{array}$ & $\begin{array}{c}\text { Durables } \\
\text { d'aventure }\end{array}$ \\
\hline $\begin{array}{l}\text { Refus d'acheter aux } \\
\text { entreprises irresponsables }\end{array}$ & 0,085 & $-0,050$ & $\mathbf{- 0 , 3 3}$ & $-0,202$ & $\mathbf{0 , 1 9 9}$ \\
Anova $: \mathrm{F}=3,85 ; \mathrm{P}=0,004$ & & & - & & + \\
\hline $\begin{array}{l}\text { Achat de produits partage } \\
\text { Anova }: \mathrm{F}=5,28 ; \mathrm{P}=0,000\end{array}$ & $\begin{array}{c}\mathbf{0 , 1 6 0} \\
+\end{array}$ & $-0,126$ & $-0,144$ & $\mathbf{- 0 , 4 0 3}$ & $\mathbf{0 , 1 9 7}$ \\
\hline $\begin{array}{l}\text { Défense du petit commerce } \\
\text { Anova F=6,12; } \mathrm{P}=0,000\end{array}$ & $\begin{array}{c}\mathbf{0 , 2 1 8} \\
+\end{array}$ & $-0,064$ & $\mathbf{- 0 , 4 3 5}$ & $-0,206$ & $\mathbf{0 , 1 9 1}$ \\
\hline $\begin{array}{l}\text { Intérêt pour origine } \\
\text { géographique }\end{array}$ & $\mathbf{0 , 2 4 7}$ & $-0,132$ & $\mathbf{- 0 , 2 5 9}$ & 0,033 & + \\
\hline Anova F=2,93; $\mathrm{P}=0,021$ & + & & - & & 0,022 \\
\hline $\begin{array}{l}\text { Réduction de son volume } \\
\text { de consommation } \\
\text { Anova F=6,93; } \mathrm{P}=0,000\end{array}$ & $\mathbf{0 , 2 2 3}$ & $\mathbf{- 0 , 0 2 3}$ & $\mathbf{- 0 , 2 6 4}$ & $\mathbf{- 0 , 4 8 9}$ & $\mathbf{0 , 1 3 9}$ \\
\hline $\begin{array}{l}\text { Score total }(=\text { somme des } \\
\text { scores moyens aux 5 facettes } \\
\text { de CSR) }\end{array}$ & $\mathbf{0 , 9 3 2 8}$ & $\mathbf{- 0 , 3 9 4}$ & $\mathbf{- 1 , 4 3 0}$ & $\mathbf{- 1 , 2 6 7}$ & $\mathbf{0 , 7 4 8}$ \\
\hline
\end{tabular}

NB. : Les moyennes en gras sont différentes avec au moins un autre sous-groupe, supérieure en gras italique et inférieure en gras 


\section{Discussion et conclusion}

Cet article avait pour objectif de présenter une typologie des consommateurs français sur le tourisme durable afin de mieux connaître la part d'individus intéressés par cette nouvelle façon de voyager et leur profil. Les analyses de données nous ont conduit à construire une échelle du tourisme durable, comprenant 5 facteurs : l'idée d'accepter de renoncer à son confort, d'être prêt à voyager avec un tour opérator responsable, de maximiser les ressources des locaux, d'accepter de voyager moins loin et enfin de protéger le capital naturel et culturel des lieux visités. Cette échelle, construite à partir de la littérature permet de mieux caractériser le tourisme durable et offre un premier outil de mesure de ce concept dans un contexte français. Les 5 dimensions obtenues semblent en effet bien refléter les contraintes du tourisme durable. Le tourisme durable est donc une nouvelle façon de voyager qui suppose de partir moins loin et de renoncer à son confort, ce qui tranche avec l'image très consensuelle et grand public de ce concept.

Ces 5 facteurs nous ont servi de base à la construction d'une typologie, faisant apparaitre 5 groupes. Deux groupes de voyageurs responsables apparaissent : les «néodurables » et les «durables d'aventure ». Les deux profils sont concernés par le tourisme durable, mais le premier semble le mieux représenter le profil du voyageur éthique car il part près de chez lui, et évite ainsi trop de pollution liée au transport aérien. Même si c'est un choix dicté par des contraintes budgétaires, ses actes correspondent parfaitement au tourisme durable pris dans un sens large, c'est-à-dire incluant également l'empreinte écologique des trajets pour se rendre sur le lieu de destination. A eux deux, ces deux groupes représentent 45,5\% de l'échantillon. Ce chiffre, s'il est enthousiasmant traduit sans doute un biais de désirabilité sociale dans les réponses. Les données démontrent malgré tout que le tourisme durable peut séduire potentiellement une grosse part des individus. Les profils non durables accordent trop d'importance à leur détente (les séjours club), à leur liberté (les campeurs) ou à leur confort (les institutionnels cultureux) pour changer leur habitudes de vacances. Les organisateurs de voyages solidaires peuvent cependant se rassurer sur l'adhésion des français au tourisme durable. Celui-ci ne semble pas réservé à une élite mais pourrait toucher un segment de voyageurs important.

Enfin, la dernière partie de l'article s'est penchée sur le lien entre tourisme durable et consommation socialement responsable. Les analyses montrent qu'il existe un lien très fort entre les deux concepts et qu'en réalité touristes durables et consommateurs responsables représentent les mêmes personnes. L'engouement pour un tourisme plus responsable n'est donc pas une mode éphémère: c'est le reflet d'attentes profondes des consommateurs concernés qui manifestent leurs préoccupations sociales et environnementales dans leur consommation quotidienne. A nouveau, les professionnels du tourisme souhaitant se positionner sur le créneau du développement durable doivent donc garder à l'esprit qu'ils visent des consommateurs avant de viser des touristes. Même dans un contexte de détente, les touristes durables restent vigilants sur leurs valeurs d'environnement et de solidarité.

Concernant les limites de ce travail, nous pouvons citer le fait de travailler sur des données issues d'autodéclaration des répondants. Les résultats obtenus surestiment certainement le phénomène du tourisme durable qui demeure encore marginal au sein de nos sociétés. Nous pouvons également remarquer que la littérature sur les touristes durables étant assez pauvre, les items de l'échelle de TD proposée s'appuient sur un petit nombre de contributions et peuvent manquer d'une certaine validité. La première version de l'échelle mériterait d'être testée sur de nouvelles données et d'être confrontée à des experts du concept.

En terme de recherches futures, nous souhaitons approfondir le lien entre CSR et TD, voire par exemple à travers une modélisation quelles dimensions de la CSR sont liées à celles du TD. En effet, dans la présente recherche, nous avons montré qu'il existe un lien entre TD et 
CSR mais nous n'avons pas pour autant montré que tous les consommateurs socialement responsables sont des touristes durables. De façon plus générale, d'avantage de recherches sont nécessaires sur le concept de tourisme durable, encore trop peu exploité dans l'univers académique. Ce concept émergent mériterait plus d'attentions d'autant que l'urgence climatique rendra ce thème toujours plus au cœur de l'actualité. 


\section{Références bibliographiques}

Babou I. et Callot P. , 2007, Les dilemmes du tourisme, Vuibert.

Becker L.J., Seligman C., Fazio R.H. \& Darley J.M., 1981, Relating attitudes to residential energy use, Environment and Behavior, 13, 5, pp. 590-609.

Béji-Bécheur A. et Bensebaa F., 2006, Companies' practices and social responsability : cases of companies in the French tourist sector, Ethics and Economics, 3, 2 (http://ethiqueeconomique.org/).

Bramwell et Lane, 2008, Priorities in sustainable tourism research, Journal of Sustainable Tourism, 16,1, 1-4.

Choi H. C. et Sirakaya E., 2005, Measuring residents attitude toward sustainable tourism : development of sustainable tourism attitude scale, Journal of Travel Research, 43,4, 380-394.

Dolnicar S., Crouch G. I. \& Long P., 2008, Environment-friendly tourists: What do we really know about them? , Journal of Sustainable Tourism, 16, 2, 197-210.

Evrard Y., Bourgeon D. \& Petr C., 2000, Le comportement de consommation culturelle : un état de l'art, $16^{\text {ème }}$ Congrès International de l'Association Française du Marketing, Montréal, Canada, Mai.

Francois-Lecompte A. (2005), La consommation socialement responsable: proposition et validation d'un cadre conceptuel intégrateur, Thèse de doctorat, Université Pierre Mendès France, Grenoble 2.

Francois-Lecompte A. et Valette-Florence P. (2006), «Mieux connaître le consommateur socialement responsable », Décisions Marketing, 41, 67-80, 2006.

Frochot I. \& Legohérel P., 2007, Le marketing du tourisme, Dunod.

Galloway G., 2002, Psychographic segmentation of park visitor markets : evidence for the utility of sensation seeking, Tourism Management, 23, 581-506.

Gössling S., Hall M., Lane B. et Weaver D., 2008, The Helsingborg Statement on Sustainable Tourism, Journal of Sustainable Tourism, 16, 1, 122-124.

Jolibert A. \& Jourdan P., 2006, Marketing Research, Dunod.

Lansing P. et De Vries P. (2006), Sustainable Tourism: Ethical Alternative or Marketing Ploy, Journal of Business Ethics, 72, 1, 77-85.

La RevueDurable, 2008, Dossier spécial « vers un tourisme de proximité, riche d'expériences fortes », Juillet-Septembre, $N^{\circ} 30$.

Liu, 2003, Sustainable tourism development : a critique, Journal of Sustainable Tourism, 11,6, 459- 475 .

Organisation Mondiale du Tourisme, 2008, Baromètre du tourisme mondial, janvier.

Palacio V. et McCool S.F., 1997, Identifying ecotourists in Belize through benefit segmentation: a preliminary analysis, Journal of Sustainable Tourism, 5, 3, 234-243.

Tenenhaus M., 2007, Statistique, Dunod.

Tregear A., McLeay F. \& Moxey A, 1997, Sustainability and Marketing: Competitive or complementary?, chapter 8, in M.J. Stabler editor, Tourism sustainability: principles to practice, CAB International, 119-127.

Weaver L.J.et Lawton L.J., 2002, Overnight ecotourist market segmentation in the gold coast Hinterland of Australia, Journal of Travel Research, 40, 3, 270-280. 